\title{
Improving Light Extraction of Organic Light-Emitting Devices by Attaching Nanostructures with Self-Assembled Photonic Crystal Patterns
}

\author{
Kai-Yu Peng and Da-Hua Wei \\ Graduate Institute of Manufacturing Technology and Department of Mechanical Engineering, \\ National Taipei University of Technology (TAIPEI TECH), Taipei 10608, Taiwan
}

Correspondence should be addressed to Da-Hua Wei; dhwei@ntut.edu.tw

Received 7 April 2014; Revised 23 June 2014; Accepted 28 June 2014; Published 20 July 2014

Academic Editor: Ching-Song Jwo

Copyright (C) 2014 K.-Y. Peng and D.-H. Wei. This is an open access article distributed under the Creative Commons Attribution License, which permits unrestricted use, distribution, and reproduction in any medium, provided the original work is properly cited.

A single-monolayered hexagonal self-assembled photonic crystal (PC) pattern fabricated onto polyethylene terephthalate (PET) films by using simple nanosphere lithography (NSL) method has been demonstrated in this research work. The patterned nanostructures acted as a scattering medium to extract the trapped photons from substrate mode of optical-electronic device for improving the overall external quantum efficiency of the organic light-emitting diodes (OLEDs). With an optimum latex concentration, the distribution of self-assembled polystyrene (PS) nanosphere patterns on PET films can be easily controlled by adjusting the rotation speed of spin-coater. After attaching the PS nanosphere array brightness enhancement film (BEF) sheet as a photonic crystal pattern onto the device, the luminous intensity of OLEDs in the normal viewing direction is $161 \%$ higher than the one without any BEF attachment. The electroluminescent (EL) spectrum of OLEDs with PS patterned BEF attachment also showed minor color offset and superior color stabilization characteristics, and thus it possessed the potential applications in all kinds of display technology and solid-state optical-electronic devices.

\section{Introduction}

Organic light-emitting diode is one of the optical-electronic devices for the various applications not only in general illumination but also for consumer displays due to the probable advantages of excellent EL performances, good flexibility, light weight, better durability, low power consumption, and convenient fabrication. Therefore, the OLEDs seem to be one of the potential candidates for the next-generation emissive display technology [1-5]. However, many research articles have reported that almost $70 \sim 80 \%$ of the photons generated inside the organic layers is confined in the OLEDs caused by three major factors: (1) the total internal reflection (TIR) occurred at the glass/air interface and organic layers/substrate (substrate and waveguiding mode); (2) the reflection loss is due to the refractive index mismatch between interfaces (Fresnel loss); (3) the emitted light is lost due to absorption and plasmonic dissipation of the metal electrode (absorption and SP mode) [6-9]. For the reduction of the waveguiding mode of the organic layers, various methods such as introducing textured surface, inserting low-index materials, and fabricating patterned nanostructures into the device have been proposed [10-13]. But the light extracted from organic layers still suffers from the light confinement of the device substrate. Using light-coupling structures onto the surface of device can help extract light suffering from the TIR at glass substrate/air interface, thus increasing the total external quantum efficiency (EQE) [14, 15]. Many researches have proposed different ways to extract the trapped light in the substrate mode, including rough or textured substrate surface, microlens array (MLA) film, diffusive particles, and silica colloidal gel [14-16]. It is worth mentioning that, due to the limitation of the standard photolithography technique, the used microlens with diameters near the wavelength of the emitted light from the OLEDs cannot be made. In the presented work, we introduced a facile and effective 


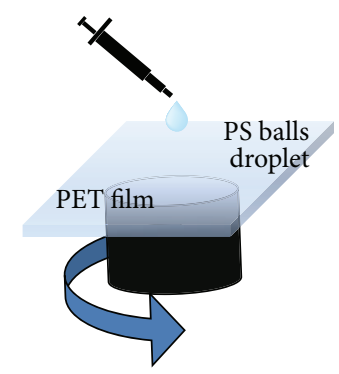

(a) PS balls spun-coated on PET thin film
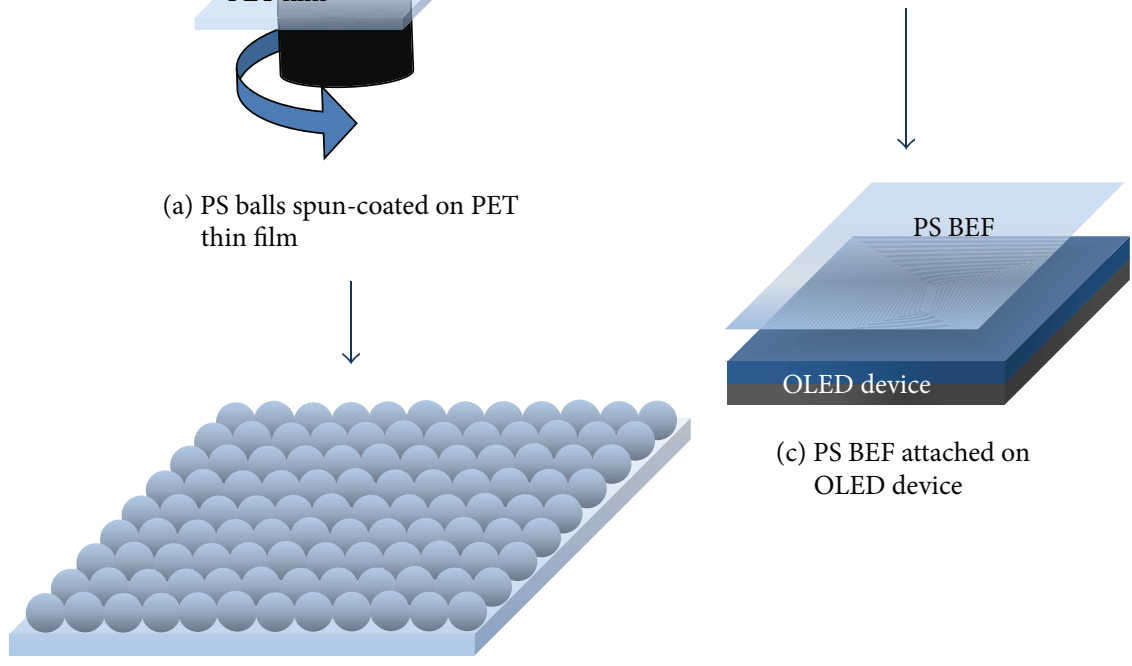

(c) PS BEF attached on OLED device

(b) Formation of PS array BEF

FIGURE 1: The schematic diagrams of (a) the fabrication procedures for (b) the preparation of single-monolayered self-assembled polystyrenes (PS) arrays, and then (c) the brightness enhancement film (BEF) and its application attached onto organic light-emitting diodes (OLEDs) devices.

fabrication method named as nanosphere lithography for the production of periodic particle array surfaces with nanometer-scale features. NSL was firstly reported by the research work of Hulteen and Van Duyne [17] and has been widely employed in the area of two- and three-dimensional (2D and 3D) micro/nanostructures patterning, such as quantum dots, nanowires, nanomesh, antireflection structure (ARS), and 3D inverse-opal photonic crystals [18-23]. Compared to those traditional sophisticated lithographic methods, NSL has shown a great many advantages that include a simple nanofabrication technique, an inexpensive route for the patterning of long-range periodic nanostructure arrays in a large scale area, and high throughput. Methods for the deposition of PS nanosphere solution onto desired substrates include electrophoretic deposition, electrochemical deposition, Langmuir-Blodgett- (L-B-) like technique, drop-coating, and spin-coating which we utilized spin-coating in our present research work [24-28].

Here, we demonstrated an unsophisticated way to effectively extract the trapped photons in the glass substrate to the outer media by attaching the PS nanosphere patterned brightness enhancement film (BEF) onto the OLEDs surface. Owing to the large increase of light extraction from trapped photons, the total luminous intensity enhancement for the device with a single-monolayered nanospheres arrangement patterned film attachment in the normal direction shows a great improvement of $161 \%$ when compared to the traditional indium tin oxide (ITO) based OLEDs without any BEF sheet attachment. The OLEDs attached with PS patterned BEF also possessed potential application in flat panel consumer display technology due to the minor color offset and good color stabilization properties in different viewing angles [29].

\section{Experimental Procedure}

Figure 1 shows the schematic diagrams of the fabrication process for the preparation of a single-monolayered PS array BEF and its application in OLED devices. At first, the methanol solution mixing with a surfactant Triton X-100 (400:1 in volume) is prepared to dilute the purchased PS beads. Then the surfactant-free nanometer-scale polystyrenes with latex colloids (Bangs Laboratories Inc., $10 \mathrm{wt} \%$ in water, PS mean diameters: ranging from 200 to $800 \mathrm{~nm}$ ) were further diluted by the above-mentioned methanol solution in ultrasonic bath. Those as-prepared diluted PS nanospheres with latex solution were spun-coated onto clean PET substrates by the spin-coater as shown in Figure 1(a). The distribution of a single-monolayered self-assembled PS nanosphere arrays can be easily controlled by adjusting the rotation speed of spincoater as shown in Figure 1(b). The period of the hexagonal patterns is determined by the initial diameter of PS beads and attaching the PS nanosphere array BEF sheet as a photonic crystal pattern onto the OLED device as shown in Figure 1(c). The product information and experimental details for the PS nanospheres with the periodicity ranging from 200 to $800 \mathrm{~nm}$ are listed in Table 1.

The commonly used techniques for fabricating the OLEDs are separated into two major types, including dry and wet-chemical processes. The wet-chemical process includes 
TABLE 1: The product information and experimental details are for the PS nanosphere with different periodicities.

\begin{tabular}{|c|c|c|c|c|c|}
\hline Diameter of PS balls (nm) & Product number & $\begin{array}{c}\text { Content of solid PS } \\
\text { in stock solution } \\
(\%)\end{array}$ & $\begin{array}{l}\text { Solution proportion } \\
\text { (methanol: PS latex) }\end{array}$ & $\begin{array}{c}\text { Concentration of PS } \\
\text { beads in prepared } \\
\text { solution (\%) }\end{array}$ & Spin speed (rpm) \\
\hline 200 & \multirow{3}{*}{ PS02N } & 10.0 & $2: 1$ & 3.33 & 2000 \\
\hline 300 & & 10.0 & $1: 1.5$ & 6.00 & 1400 \\
\hline 400 & & 9.7 & $1: 1.5$ & 5.82 & 1100 \\
\hline 500 & \multirow{4}{*}{ PS03N } & 10.1 & $1: 2$ & 6.73 & 800 \\
\hline 600 & & 10.5 & $1: 2$ & 7.00 & 600 \\
\hline 700 & & 9.9 & $1: 2$ & 6.60 & 500 \\
\hline 800 & & 10.1 & $1: 2$ & 6.73 & 400 \\
\hline
\end{tabular}

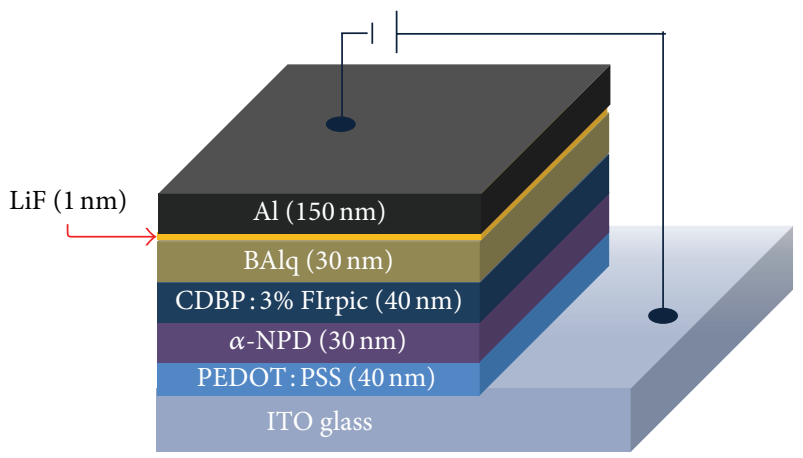

(a)
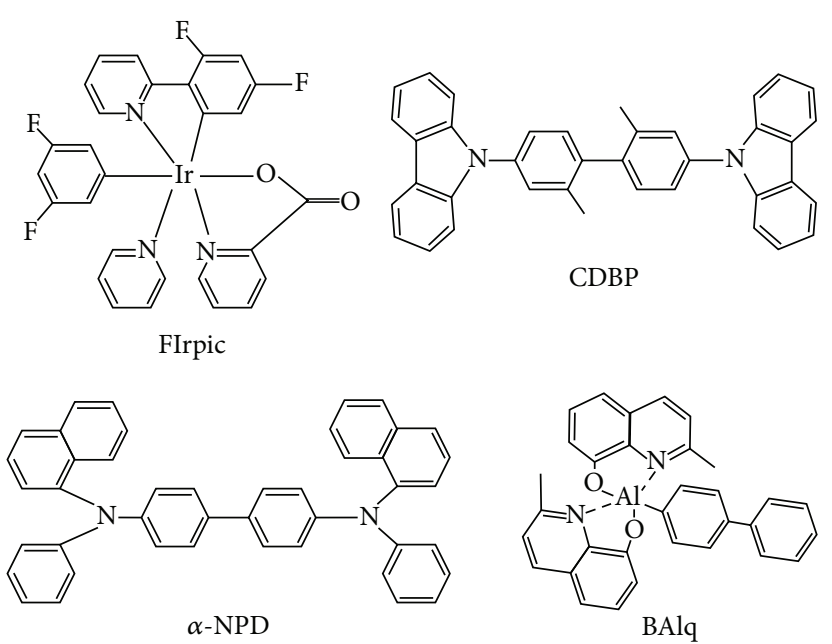

(b)

FIGURE 2: (a) The diagram is for the OLEDs device structure, and (b) the chemical structures of the molecules employed in the presented research work.

the spin-coating and inkjet-printing methods, which is suitable for the fabrication of the polymer OLEDs [30, 31]. However, the dry process (vacuum evaporation deposition) applied in our work is mainly used to fabricate the small molecule OLEDs [15]. In the parts of the blue phosphorescent OLED fabrication process, the FIrpic-based light-emitting diode was made onto an ITO coated glass substrate (ITO film thickness: $200 \mathrm{~nm}$, sheet resistance: $10 \Omega / \square$ ). Prior to the organic layers evaporation process, the ITO glass was thoroughly cleaned with acetone, isopropyl alcohol, ethanol, and deionized water in sequence and then treated with an UV-ozone cleaner. The multilayers structure for the OLEDs is depicted in Figure 2(a). The $40 \mathrm{~nm}$ thick poly(3,4-ethylenedioxythiophene) : poly(4-styrenesulfonate) (PEDOT : PSS) thin films were spun onto the ITO coated glass to reduce the possibility of electrical shorts. The organic layers were continuously deposited onto the substrates in an ultrahigh vacuum (around $5 \times 10^{-6}$ torr) by thermal evaporation deposition system. The hole-transporting layer $\left(\mathrm{N}, \mathrm{N}^{\prime}\right.$-diphenyl-N,N ${ }^{\prime}$ bis(1-naphthyl)-(1,1'-biphenyl)- $4,4^{\prime}$-diamine, $\alpha$-NPD), blue emissive layer (CDBP:3\% FIrpic), electron-transporting, and hole-blocking layer (aluminum (III) bis(2-methyl-8quninolinato)-4-phenylphenolate, BAlq) were sequentially evaporated onto the substrates. Then $\mathrm{LiF}$ and thick Al were set as the opaque cathode for the OLED device. The detailed device structure is composed of ITO $(200 \mathrm{~nm}) / \mathrm{PEDDT}$ : PSS $(40 \mathrm{~nm}) / \alpha$-NPD $(30 \mathrm{~nm}) / \mathrm{CDBP}: 3 \%$ FIrpic $(40 \mathrm{~nm}) / \mathrm{BAlq}$ $(40 \mathrm{~nm}) / \mathrm{LiF}(1 \mathrm{~nm}) / \mathrm{Al}(150 \mathrm{~nm})$. Figure $2(\mathrm{~b})$ shows the molecule structures of $\alpha$-NPD, CDBP, FIrpic, and BAlq, respectively. The surface morphology was observed by field emission scanning electron microscope (FESEM, Hitachi S4800). In order to prepare the SEM specimens, the dilute PS nanosphere latex was spun-coated on the flat PET film thus forming a single-layered close-packed PS array at first. Then the as-prepared samples were put into a vacuum sputtering system to deposit $5 \mathrm{~nm}$ Au film. The conductivity of those samples is increased and the point discharge effect would be decreased. Finally, those above-mentioned SEM samples were cut into two pieces for the preparation of the SEM cross-sectional analysis. The EL spectrum, currentvoltage characteristics, and luminance for optical-electronic devices were examined by a measuring system consisting of 
TABLE 2: The distribution results for the self-assembled PS nanosphere patterns at different spin speed conditions.

\begin{tabular}{lcccc}
\hline Diameter of PS balls (nm) & $\begin{array}{c}\text { Concentration } \\
\text { (methanol: PS latex) }\end{array}$ & Spin speed (rpm) & Corresponding image & Results \\
\hline 400 & $1: 2$ & 2000 & Figure 3(a) & $\begin{array}{l}\text { Random arranged PS } \\
\text { patterns with obvious } \\
\text { defects }\end{array}$ \\
\hline 400 & $1: 2$ & 500 & Figure 3(b) & $\begin{array}{l}\text { Single- or multilayered PS } \\
\text { balls stacking }\end{array}$ \\
\hline 400 & $1: 2$ & 1100 & Figures 3(c) and 3(d) & $\begin{array}{l}\text { Single-layered and } \\
\text { close-packed PS patterns }\end{array}$ \\
\hline
\end{tabular}

a spectrophotometer (Minolta CS-1000) connecting with a computer and a power supply unit (Keithley model 2400) to drive the OLEDs. The angular dependence of the EL intensity was measured by putting the OLEDs device vertically on the center of the rotation stage.

\section{Results and Discussion}

In the typical spin-coating process, the PS balls followed the lowest energy level rule of self-assembly and rearranged onto any kinds of the substrates freely till the solution was dried. Following the lowest energy level rule of selfassembly, a single-monolayered hexagonal closed-packed structure with uniform distribution is formed onto the PET films after few minutes. The period of this hexagonal pattern was determined by the diameter values of PS nanospheres. Since the assembly process occurs during the drying step, two parameters including the colloid latex concentration and rotation speed of spin-coater both play a main role in achieving the monolayer hexagonal and closed-packed PS patterns over the large areas. With a specified latex concentration, the distribution of PS nanosphere patterns on PET films can be easily controlled by simply adjusting the rotation speed. Figures 3(a)-3(c) show the SEM images for $400 \mathrm{~nm}$ PS nanospheres spun-coated at different rotation speed conditions after the self-assembly process. The rapid evaporation and stronger centrifugal force occurred at a spin speed of $2000 \mathrm{rpm}$ resulting in the obvious empty space between nanoparticle islands because there is no enough time for those PS nanospheres rearranging into well-ordered 2D islands as shown in Figure 3(a). On the other hand, if the spin speed was reduced to a lower value such as $500 \mathrm{rpm}$, the slow evaporation rate and weak centrifugal force would lead the consequence into randomly distributed single- or multilayered PS balls stacking as shown in Figure 3(b). Figure 3(c) shows that a single-monolayered close-packed PS pattern formed onto PET sheet in a large area can be achieved by tuning the rotation speed of spin-coater to a proper value, such as $1100 \mathrm{rpm}$ for $400 \mathrm{~nm}$ PS nanospheres in the presented work. Figure 3(d) shows the enlarged SEM image of a singlelayered close-packed $400 \mathrm{~nm}$ period PS nanosphere patterns enlarged from Figure 3(c), and the inset image is the corresponding cross-sectional morphology. Figure 3 also shows the SEM images for (e) 200 and (f) $800 \mathrm{~nm}$ PS nanospheres arrays spun-coated at rotation speed of 2000 and $400 \mathrm{rpm}$, respectively. The distribution results for self-assembled PS nanosphere patterns with $400 \mathrm{~nm}$ periodicity at different spin speed parameters are summarized and listed in Table 2.

The emission peak for the used OLED device in the presented work is located at the wavelength of $466 \mathrm{~nm}$ and with a subpeak at $497 \mathrm{~nm}$ as shown in Figure 4(a), which agreed with the work reported by Tokito et al. [32]. The turnon voltage and the saturated current efficiency of the OLED device at the current density of $10 \mathrm{~mA} / \mathrm{cm}^{2}$ are $8.7 \mathrm{~V}$ and $7.0 \mathrm{~cd} / \mathrm{A}$, respectively, as shown in Figure 4(b).

The schematic diagram as depicted in Figure 5(a) illustrated that most of the emitted light generated from the organic layer was confined in the device because of the waveguiding effect and substrate mode. In general, less than one-third of the generated photons can escape from emitting devices in typical OLEDs. One-third of these photons are guided in the glass substrate and the others are trapped in the organic layers. The photons trapped in the glass substrate can be further coupled out when applying a patterned BEF sheet attached onto OLEDs as shown in Figure 5(a). Using the coupling equation can help to understand the coupling of light in the optical-electronic device. The diffraction angle $\left(\theta_{b}\right)$ for the light trapped in the substrate is calculated by

$$
\frac{2 \pi}{\lambda_{0}} n_{b} \sin \theta_{b} \pm m \frac{2 \pi}{\Lambda}=k_{\text {glass }}=\frac{2 \pi}{\lambda_{0}} n_{b},
$$

where $m=1$ (the diffraction order), $k_{\text {glass }}$ is the propagation constant in the glass, $n_{b}=1.52$ for a typical BK7 glass, $\lambda_{0}=466 \mathrm{~nm}$, and $\Lambda=400 \mathrm{~nm}$ (the periodicity of the PS nanospheres) in this case. $\theta_{b}$ is $13.6^{\circ}$ smaller than the refraction angle in the air $\left(\theta=20.9^{\circ}\right.$, calculated by Snell's law) demonstrating the trapped photons can escape from glass to air. The insets in Figure 5(b) show the optical images of OLEDs with and without 2D PS nanosphere patterned film attachment. It reveals that the overall luminous intensity of OLED device with 2D PS photonic crystal patterned film attachment is higher than the referenced device. The PS patterned film acted as a BEF to help in extracting trapped photons in the glass/air interface to the outside. The luminous enhancement of OLED devices attached with 2D photonic crystal patterned films of various nanosphere diameters (from 200 to $800 \mathrm{~nm}$ ) is also shown in Figure 5(b). It can be seen that the luminous enhancement is increased with the decrease of the PS nanosphere diameter values for 


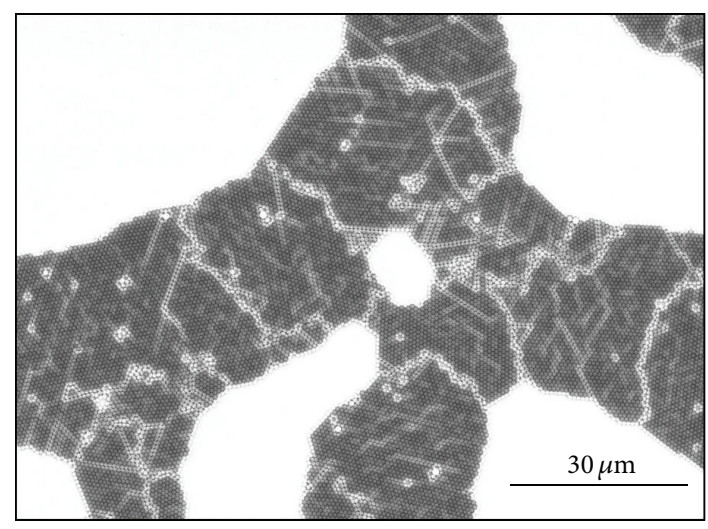

(a)

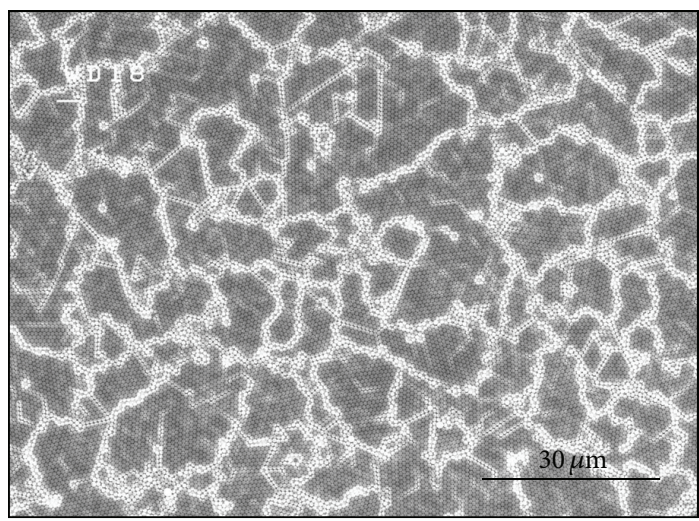

(c)

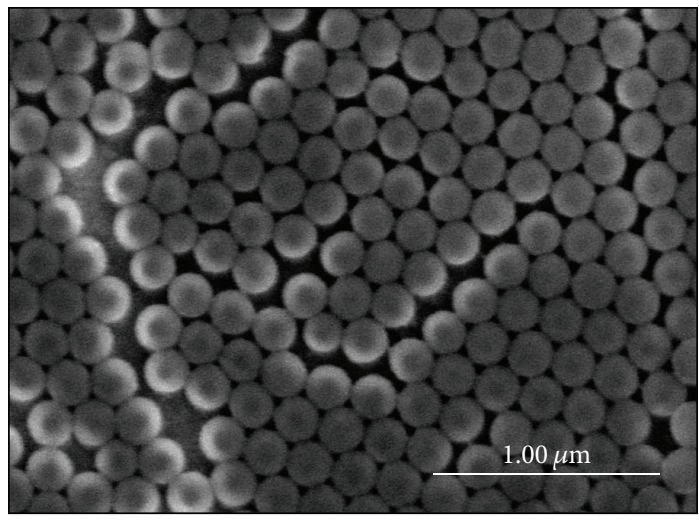

(e)

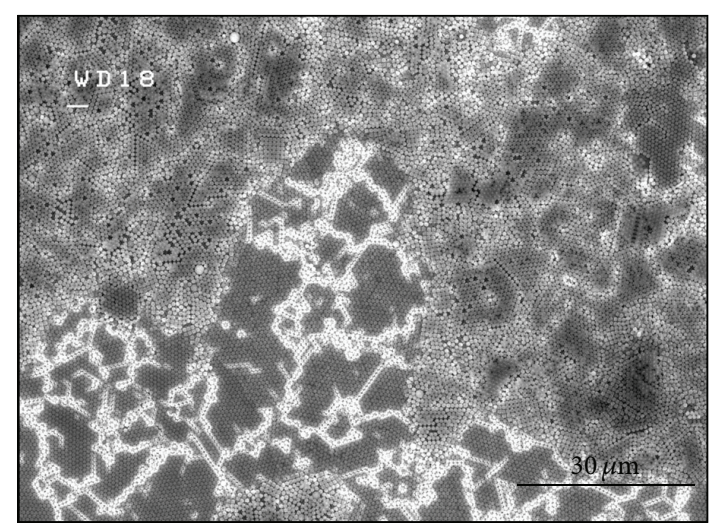

(b)

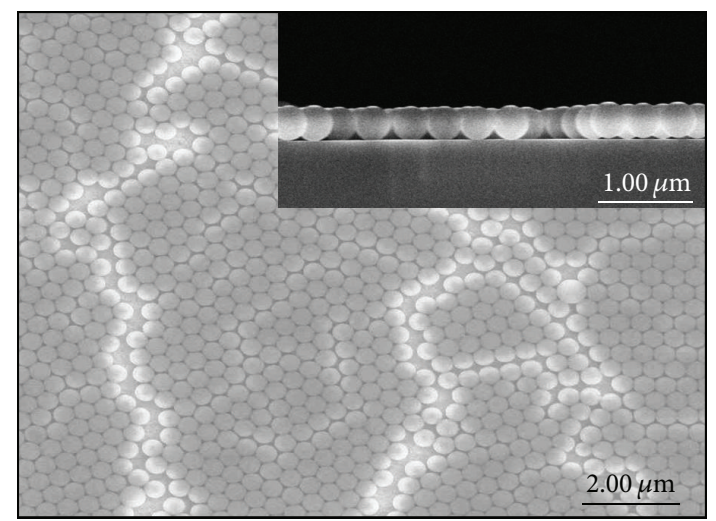

(d)

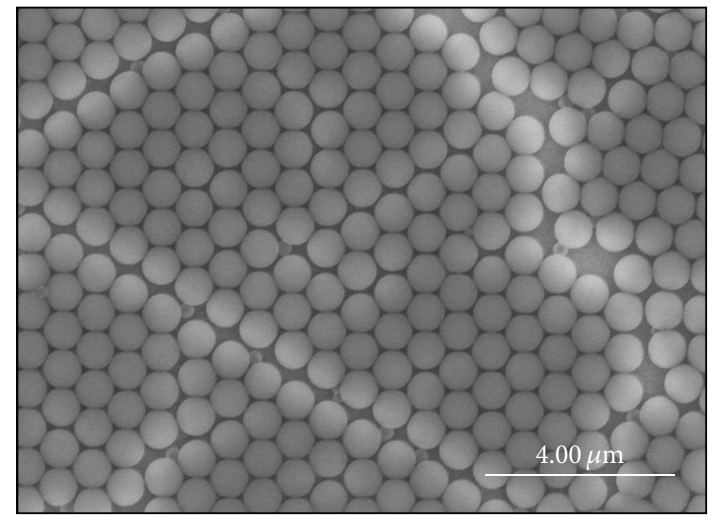

(f)

FIGURE 3: SEM images are for the $400 \mathrm{~nm}$ period PS nanospheres distribution at spin speed of (a) $2000 \mathrm{rpm}$, (b) $500 \mathrm{rpm}$, and (c) $1100 \mathrm{rpm}$, respectively. (d) The enlarged SEM image is from (c) for a single-monolayered nanosphere array patterns with hexagonal close-packed uniform distribution and the inset image is the corresponding cross-sectional morphology. SEM images are for PS nanospheres arrays with (e) 200 and (f) $800 \mathrm{~nm}$ periodicity at spin speed of 2000 and $400 \mathrm{rpm}$, respectively.

the photonic crystal patterns with the periodicity of $400-$ $800 \mathrm{~nm}$. According to the diffraction term $(2 \pi / \Lambda)$ as shown in the grating coupling equation, the PS balls with $400 \mathrm{~nm}$ diameter (e.g., $\Lambda=400 \mathrm{~nm}$ ) show the greatest luminous enhancement efficiency and the total luminous enhancement for the device with applying PS nanosphere arrangement patterned film attachment shows a great improvement of $61 \%$ when compared to the traditional ITO-OLEDs without any
BEF attachment owing to the large increase of light extraction from trapped photons. The luminance enhancement factor then drops as the PS diameters in the range between 200 and $300 \mathrm{~nm}$. The results can be concluded into two reasons: (1) the scattering effect of the PS ball pattern is decreased because the diameter values of the PS are smaller than the wavelength of visible light; (2) the diffraction term $(2 \pi / \Lambda)$ is increasing as the PS diameters decrease; therefore, 


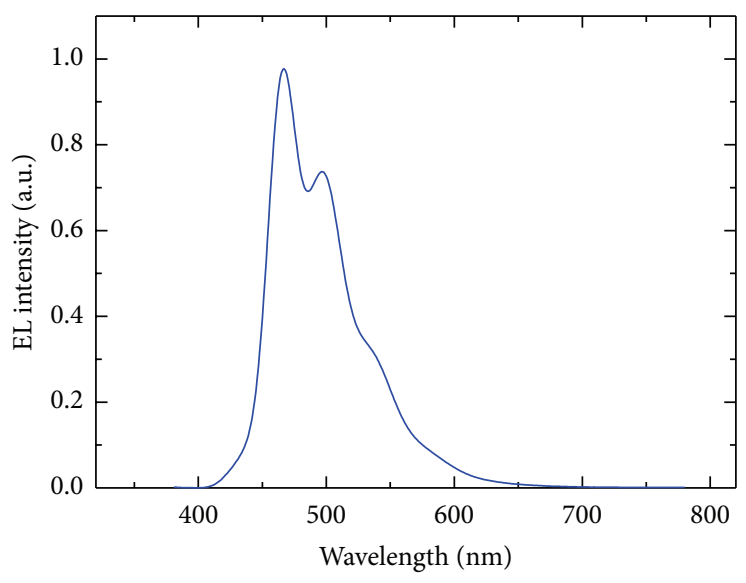

(a)

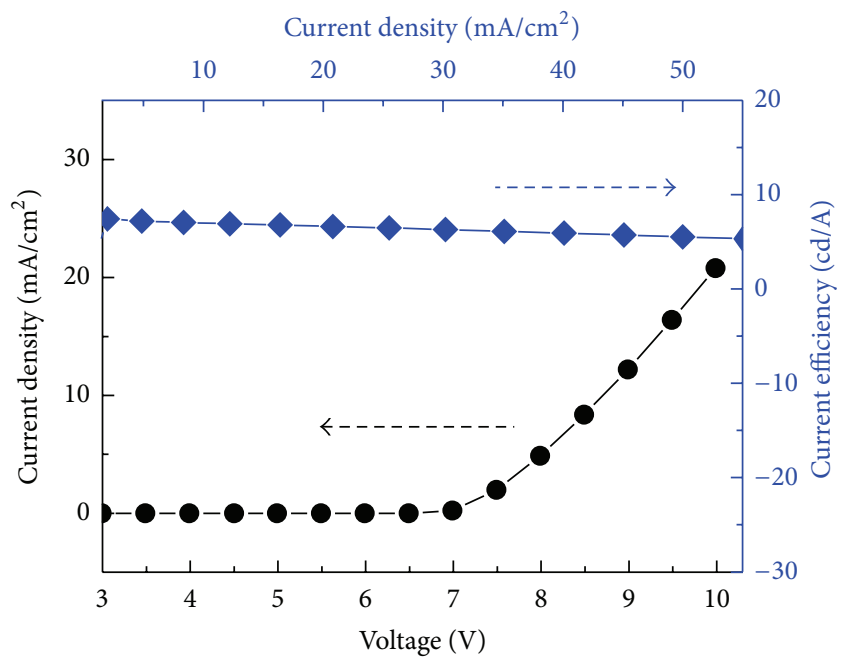

(b)

FIgUre 4: (a) EL spectrum of the OLED device. (b) $J$ - $V$ characteristic and current efficiency versus current density of the OLED device.

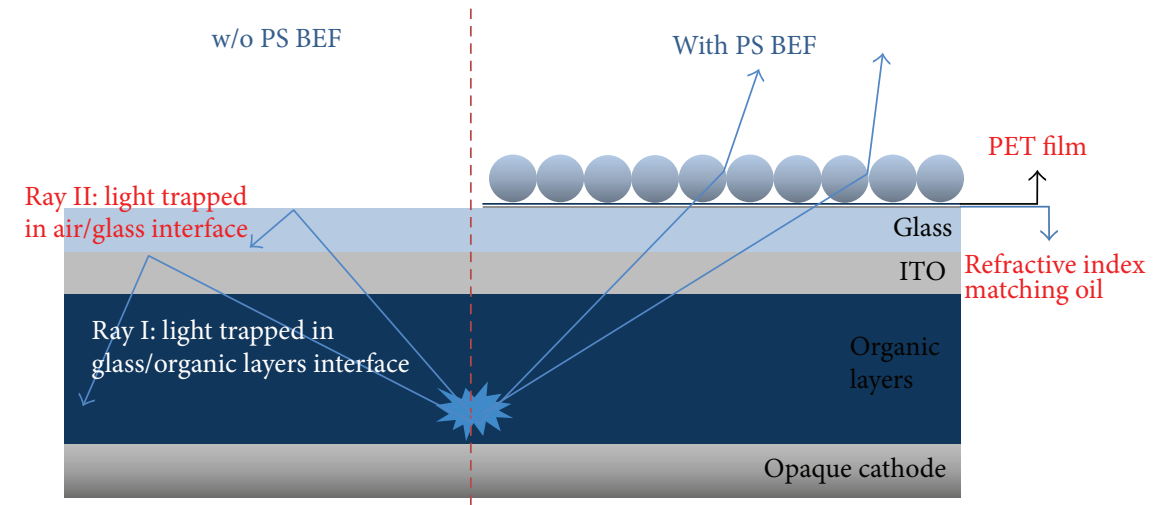

(a)

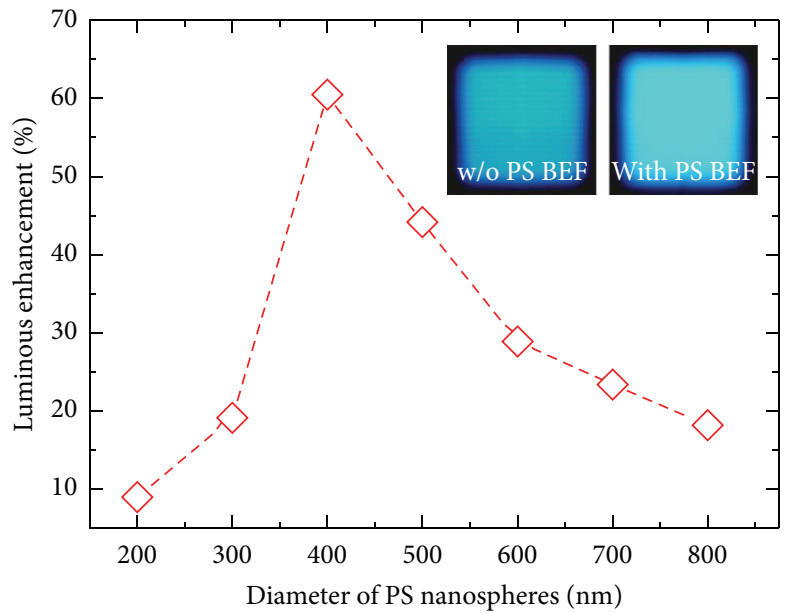

(b)

FIGURE 5: (a) Light extraction of trapped photons in glass substrate and organic layer of bottom emission OLED and (b) luminous enhancement of OLED devices attached with 2D photonic crystal pattern structures of various nanosphere diameters ranging from 200 to $800 \mathrm{~nm}$. 


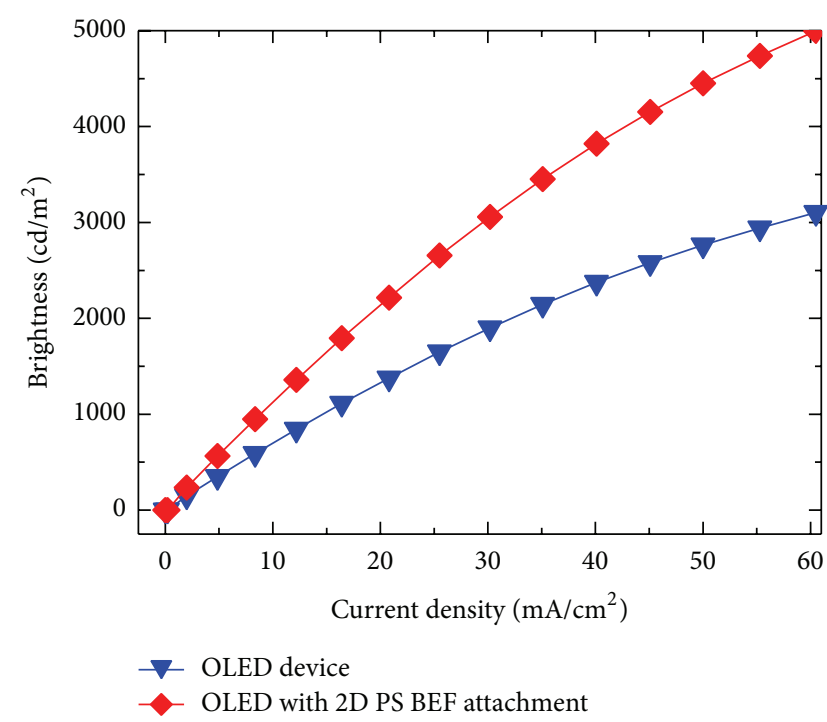

(a)

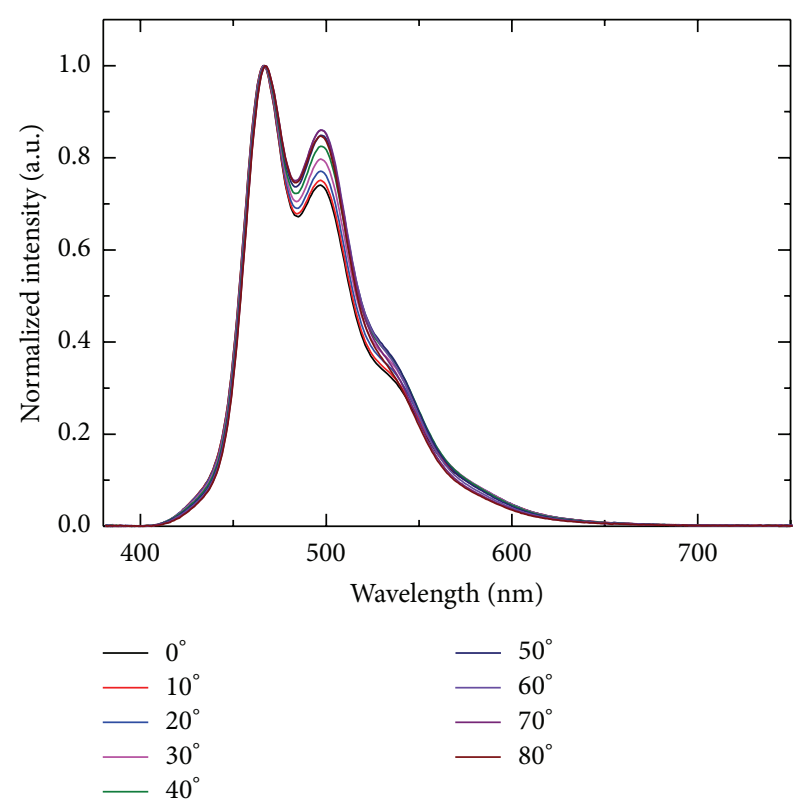

(c)

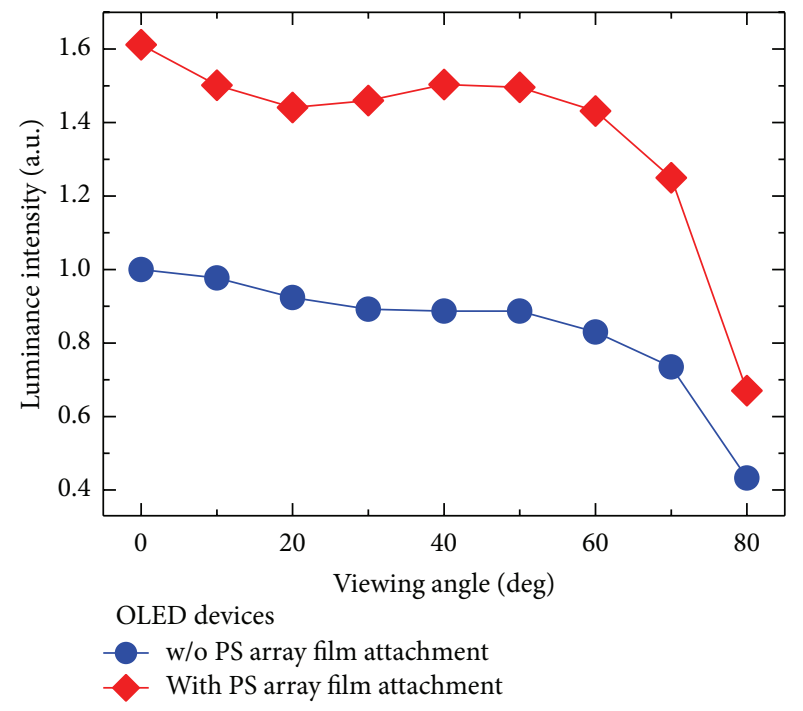

(b)

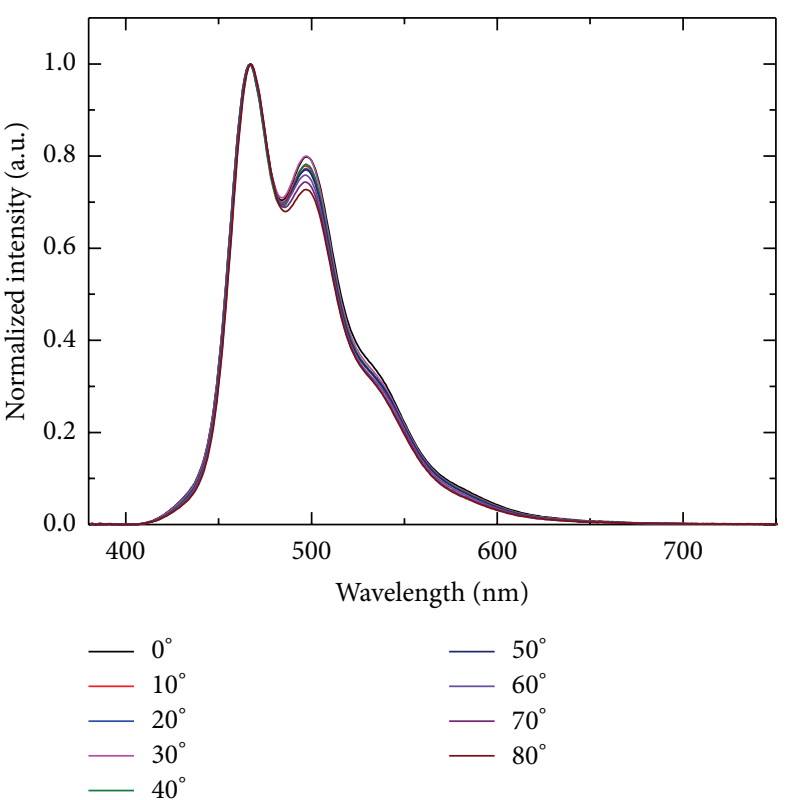

(d)

FIGURE 6: (a) Luminance versus current density and (b) the luminous intensity as a function of viewing angle for the OLED device with and without PS patterned BEF attachment. The EL spectrum for the OLED device (c) without and (d) with attaching 2D PS array patterned films (diameter: $400 \mathrm{~nm}$ ).

the calculated diffraction angle would be negative, hence lowering the scattering effect caused by the PS nanosphere patterns.

We further measured the emission light at different viewing angle (VA) to confirm the light extraction enhancement efficiency. Figure 6(a) shows the luminance values for the OLEDs with and without PS nanosphere patterned BEF sheet attachment under $30 \mathrm{~mA} / \mathrm{cm}^{2}$ operating current density in normal viewing angle that are about 3050 and $1900 \mathrm{~cd} / \mathrm{m}^{2}$, respectively. The brightness improvement is about $60.5 \%$, which is in good agreement with the result of luminous enhancement for $400 \mathrm{~nm}$ PS patterned BEF sheet attaching onto OLEDs as shown in Figure 5(b). The luminance intensity of device as a function of viewing angle with a constant current injection $\left(60 \mathrm{~mA} / \mathrm{cm}^{2}\right)$ is shown in Figure 6(b). The luminance intensity of device without PS patterned BEF attachment drops as the viewing angle increases. When we applied a patterned BEF sheet onto OLEDs, the angular emission pattern was broadened and the luminance intensity was also increased. Figure 6(c) shows the normalized EL spectrum for OLEDs without attaching PS patterned BEF sheet. The normalized intensity in the wavelength of $497 \mathrm{~nm}$ 
was changed from $0.741\left(\mathrm{VA}=0^{\circ}\right)$ to $0.849\left(\mathrm{VA}=50^{\circ}\right)$. It revealed an obvious color offset property in the wavelength of $497 \mathrm{~nm}$ at different viewing direction. But the color offset phenomenon can be minimized after applying the PS patterned BEF as shown in Figure 6(d). The normalized intensity was slightly shifted from $0.799\left(\mathrm{VA}=0^{\circ}\right)$ to $0.771\left(\mathrm{VA}=50^{\circ}\right)$. The reason for the minor color offset of the OLEDs attached with PS BEF sheet is attributed to the omnidirectional light harvesting enhancement, which is consistent with the result as shown in Figure 6(b). The emission spectrum also shows that there is almost no wavelength shift when utilizing such a PS patterned BEF attachment, which performs excellent color stabilization characteristics. The results can be confirmed by the Commission Internationale de L'Eclairage coordinates (CIE 1931) and the corresponding CIE coordinates calculated from the EL spectrum for OLEDs without and with PS patterned BEF attachment are $(0.1635,0.2798)$ and $(0.1619$, $0.2776)$, respectively.

\section{Conclusions}

In summary, using an external patterned BEF sheet to efficiently improve the total OLED's luminous efficiency has been successfully demonstrated by a facile NSL technique in the presented research work. With a specified latex concentration, the distribution of PS arrays on PET films can be easily controlled by only adjusting the rotation speed of spin-coater. The luminous intensity of OLEDs with attaching PS patterned sheet in the normal viewing direction is $161 \%$ higher than the one without any BEF attachment. The presented experimental results indicated that the trapped photons are further coupled out from the substrate mode, thus increasing the light extraction efficiency. The EL spectrum of OLEDs with PS patterned BEF attachment shows minor color offset and outstanding color stabilization property that possess potential future applications in all kinds of opticalelectronic display devices and solid-state lighting technology.

\section{Conflict of Interests}

The authors declare that there is no conflict of interests regarding the publication of this paper.

\section{Acknowledgment}

The authors acknowledge financial support of the main research projects of the National Science Council of Taiwan under Grant nos. NSC 101-2221-E-027-042 and NSC 101-2622E-027-003-CC2, respectively.

\section{References}

[1] Y. Luo, L. Wang, Y. Ding, L. Li, and J. Shi, "High lightextracting efficiency for OLED directly fabricated on doubleside nanotextured silica substrate," Optics Letters, vol. 38, no. 14, pp. 2394-2396, 2013.

[2] J. Huang, G. Li, E. Wu, Q. Xu, and Y. Yang, "Achieving highefficiency polymer white-light-emitting devices," Advanced Materials, vol. 18, no. 1, pp. 114-117, 2006.
[3] C. W. Tang and S. A. Vanslyke, "Organic electroluminescent diodes," Applied Physics Letters, vol. 51, no. 12, pp. 913-915, 1987.

[4] S. R. Forrest, D. D. C. Bradley, and M. E. Thompson, "Measuring the efficiency of organic light-emitting devices," Advanced Materials, vol. 15, no. 13, pp. 1043-1048, 2003.

[5] B. Niesen and B. P. Rand, "Thin film metal nanocluster lightemitting devices," Advanced Materials, vol. 26, no. 9, pp. 14461449, 2014.

[6] J. B. Kim, J. H. Lee, C. K. Moon, S. Y. Kim, and J. J. Kim, "Highly enhanced light extraction from surface plasmonic loss minimized organic light-emitting diodes," Advanced Materials, vol. 25, no. 26, pp. 3571-3577, 2013.

[7] S. Nowy, B. C. Krummacher, J. Frischeisen, N. A. Reinke, and W. Brütting, "Light extraction and optical loss mechanisms in organic light-emitting diodes: Influence of the emitter quantum efficiency," Journal of Applied Physics, vol. 104, no. 12, Article ID 123109, 2008.

[8] R. Meerheim, M. Furno, S. Hofmann, B. Lüssem, and K. Leo, "Quantification of energy loss mechanisms in organic lightemitting diodes," Applied Physics Letters, vol. 97, no. 25, Article ID 253305, 2010.

[9] S. Y. Kim and J. J. Kim, "Outcoupling efficiency of organic light emitting diodes and the effect of ITO thickness," Organic Electronics: Physics, Materials, Applications, vol. 11, no. 6, pp. 1010-1015, 2010.

[10] I. Schnitzer, E. Yablonovitch, C. Caneau, T. J. Gmitter, and A. Scherer, "30\% external quantum efficiency from surface textured, thin-film light-emitting diodes," Applied Physics Letters, vol. 63, no. 16, pp. 2174-2176, 1993.

[11] M. Slootsky and S. R. Forrest, "Enhancing waveguided light extraction in organic LEDs using an ultra-low-index grid," Optics Letters, vol. 35, no. 7, pp. 1052-1054, 2010.

[12] D. H. Wei, W. H. Liao, and K. Y. Peng, "Light guide of au nanostructures for color-filterness optoelectronic display devices," Journal of Nanoscience and Nanotechnology, vol. 12, no. 2, pp. 1341-1343, 2012.

[13] Y. Sun and S. R. Forrest, "Enhanced light out-coupling of organic light-emitting devices using embedded low-index grids," Nature Photonics, vol. 2, no. 8, pp. 483-487, 2008.

[14] S. Möller and S. R. Forrest, "Improved light out-coupling in organic light emitting diodes employing ordered microlens arrays," Journal of Applied Physics, vol. 91, no. 5, pp. 3324-3327, 2002.

[15] Y. H. Ho, K. Y. Chen, K. Y. Peng, M. C. Tsai, W. C. Tian, and P. K. Wei, "Enhanced light out-coupling of organic light-emitting diode using metallic nanomesh electrodes and microlens array," Optics Express, vol. 21, no. 7, pp. 8535-8543, 2013.

[16] T. Tsutsui, M. Yahiro, H. Yokogawa, K. Kawano, and M. Yokoyama, "Doubling coupling-out efficiency in organic lightemitting devices using a thin silica aerogel layer," Advanced Materials, vol. 13, no. 15, pp. 1149-1152, 2001.

[17] J. C. Hulteen and R. P. Van Duyne, "Nanosphere lithography: a materials general fabrication process for periodic particle array surfaces," Journal of Vacuum Science \& Technology A, vol. 13, no. 3, pp. 1553-1558, 1995.

[18] Y. H. Ho, K. Y. Chen, S. W. Liu, Y. T. Chang, D. W. Huang, and P. K. Wei, "Transparent and conductive metallic electrodes fabricated by using nanosphere lithography," Organic Electronics, vol. 12, no. 6, pp. 961-965, 2011. 
[19] A. Kosiorek, W. Kandulski, H. Glaczynska, and M. Giersig, "Fabrication of nanoscale rings, dots, and rods by combining shadow nanosphere lithography and annealed polystyrene nanosphere masks," Small, vol. 1, no. 4, pp. 439-444, 2005.

[20] S. Ji, J. Park, and H. Lim, "Improved antireflection properties of moth eye mimicking nanopillars on transparent glass: flat antireflection and color tuning," Nanoscale, vol. 4, no. 15, pp. 4603-4610, 2012.

[21] C. Li, G. Hong, and L. Qi, "Nanosphere lithography at the gas/liquid interface: a general approach toward free-standing high-quality nanonets," Chemistry of Materials, vol. 22, no. 2, pp. 476-481, 2010.

[22] S. B. Kim, W. W. Lee, J. Yi, W. I. Park, J. S. Kim, and W. T. Nichols, "Simple, large-scale patterning of hydrophobic $\mathrm{ZnO}$ nanorod arrays," ACS Applied Materials and Interfaces, vol. 4, no. 8, pp. 3910-3915, 2012.

[23] J. J. Dong, X. W. Zhang, Z. G. Yin et al., "Controllable growth of highly ordered $\mathrm{ZnO}$ nanorod arrays via inverted self-assembled monolayer template," ACS Applied Materials and Interfaces, vol. 3, no. 11, pp. 4388-4395, 2011.

[24] A. Winkleman, B. D. Gates, L. S. McCarty, and G. M. Whitesides, "Directed self-assembly of spherical particles on patterned electrodes by an applied electric field," Advanced Materials, vol. 17, no. 12, pp. 1507-1511, 2005.

[25] J. Aizenberg, P. V. Braun, and P. Wiltzius, "Patterned colloidal deposition controlled by electrostatic and capillary forces," Physical Review Letters, vol. 84, no. 13, article 2997, 2000.

[26] N. D. Denkov, O. D. Velev, P. A. Kralchevsky, I. B. Ivanov, H. Yoshimura, and K. Nagayama, "Mechanism of formation of two-dimensional crystals from latex particles on substrates," Langmuir, vol. 8, no. 12, pp. 3183-3190, 1992.

[27] A. S. Dimitrov and K. Nagayama, "Continuous convective assembling of fine particles into two-dimensional arrays on solid surfaces," Langmuir, vol. 12, no. 5, pp. 1303-1311, 1996.

[28] D. Wang and H. Möhwald, "Rapid fabrication of binary colloidal crystals by stepwise spin-coating," Advanced Materials, vol. 16, no. 3, pp. 244-247, 2004.

[29] L. Wang, Y. Jiang, J. Luo et al., "Highly efficient and color-stable deep-blue organic light-emitting diodes based on a solutionprocessible dendrimer," Advanced Materials, vol. 21, no. 47, pp. 4854-4858, 2009.

[30] T. R. Hebner, C. C. Wu, D. Marcy, M. H. Lu, and J. C. Sturm, "Ink-jet printing of doped polymers for organic light emitting devices," Applied Physics Letters, vol. 72, no. 5, pp. 519-521, 1998.

[31] J. P. J. Markham, S.-C. Lo, S. W. Magennis, P. L. Burn, and I. D. W. Samuel, "High-efficiency green phosphorescence from spincoated single-layer dendrimer light-emitting diodes," Applied Physics Letters, vol. 80, no. 15, pp. 2645-2647, 2002.

[32] S. Tokito, T. Iijima, Y. Suzuri, H. Kita, T. Tsuzuki, and F. Sato, "Confinement of triplet energy on phosphorescent molecules for highly-efficient organic blue-light-emitting devices," Applied Physics Letters, vol. 83, no. 3, pp. 569-571, 2003. 

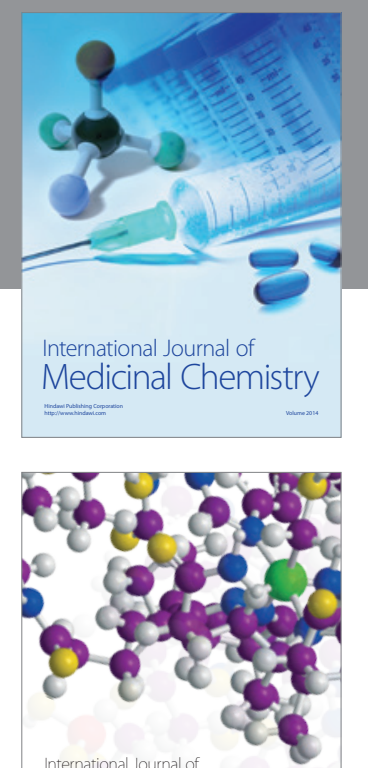

\section{Carbohydrate} Chemistry

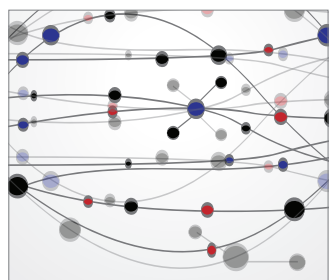

The Scientific World Journal
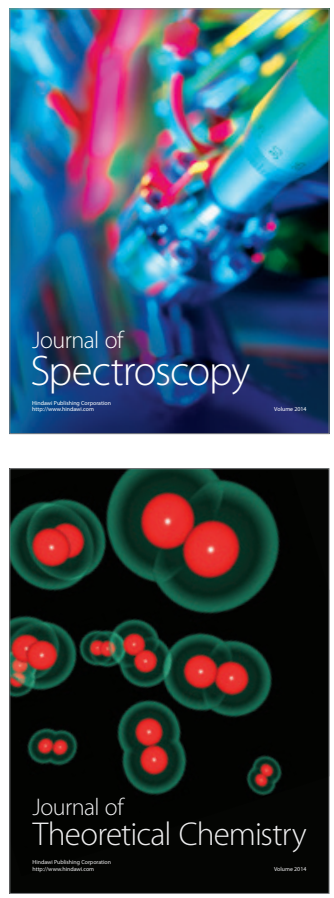
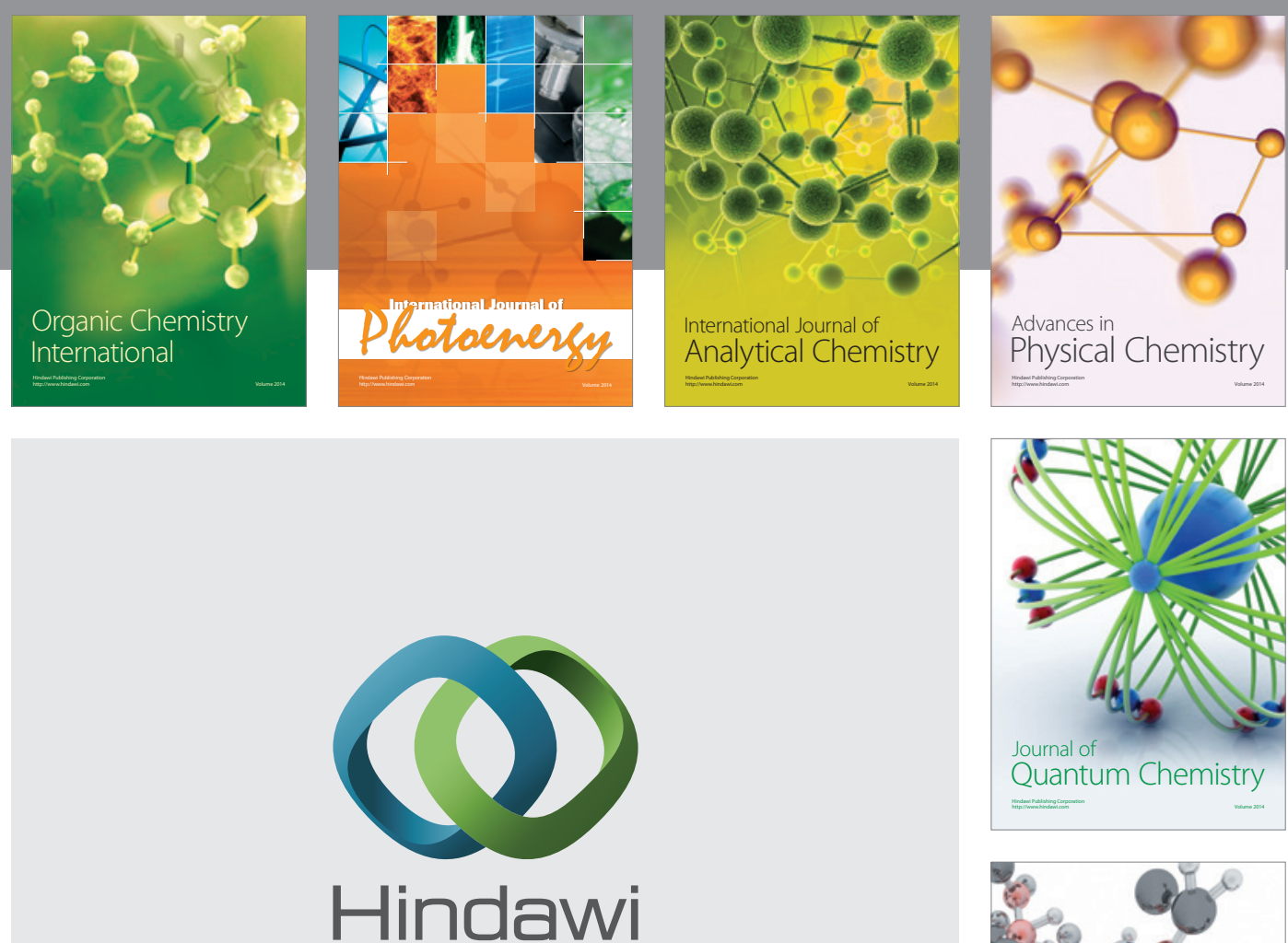

Submit your manuscripts at

http://www.hindawi.com

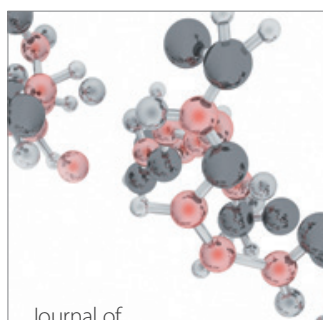

Analytical Methods

in Chemistry

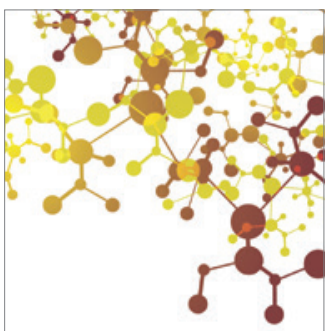

Journal of

Applied Chemistry

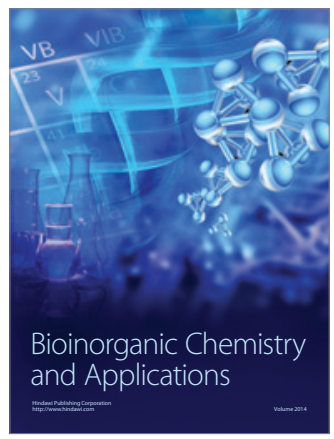

Inorganic Chemistry
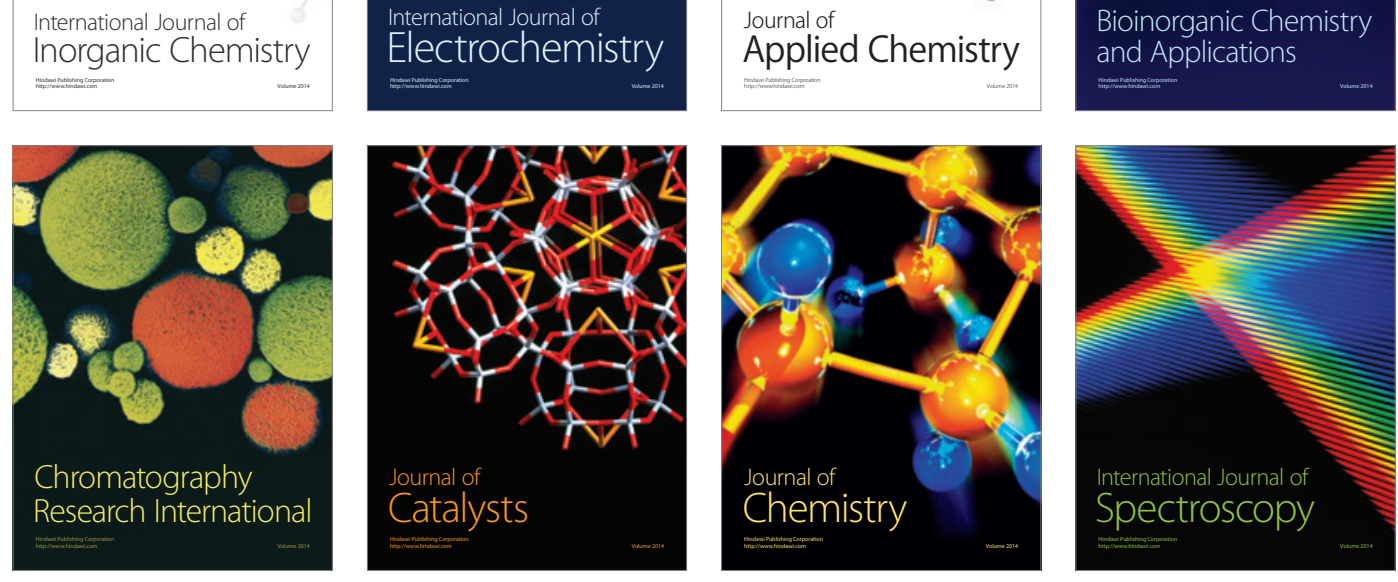